\title{
CORRECTION
}

\section{Correction to: MicroRNA in vivo precipitation identifies miR- 151-3p as a computational unpredictable miRNA to target Stat3 and inhibits innate IL-6 production}

Xiang Liu, Xiaoping Su, Sheng Xu, Huamin Wang, Dan Han, Jiangxue Li, Mingyan Huang (D) and Xuetao Cao

Cellular \& Molecular Immunology (2020) 17:110; https://doi.org/10.1038/s41423-019-0336-8

Correction to: Cellular \& Molecular Immunology (2018) 15, 99-110; https://doi.org/10.1038/cmi.2017.82, published online 11 September 2017

In the published version of Fig. 5, the bands of Stat3 in Fig. 5a and Fig. $5 \mathrm{~d}$ were exchanged by mistake, and the band of $\beta$-actin in Fig. $5 \mathrm{~b}$ was mistakenly presented with incorrected image. Figure
$5 a, 5 b$ and $5 d$ have now been corrected. The corrected version of Fig. 5 is shown below.

Although we regret our mistake during figure assembly and would like to apologize for any inconvenience it may have caused, we did not manipulate our data in any way. This unintentional error also has no bearing on the work's scientific conclusions. Corrected Fig. 5:
A

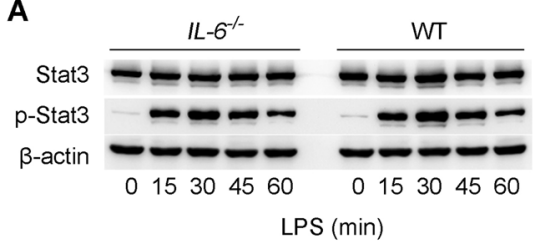

C

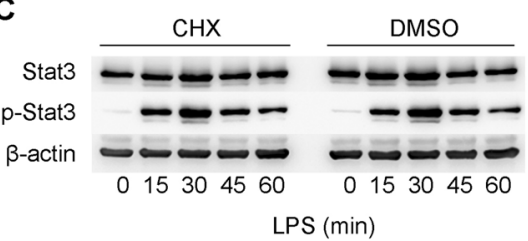

E

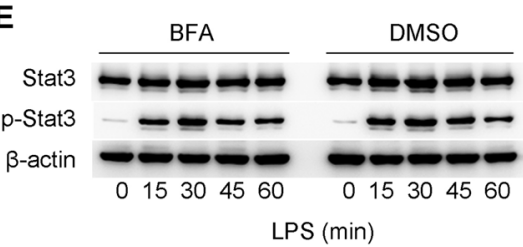

B

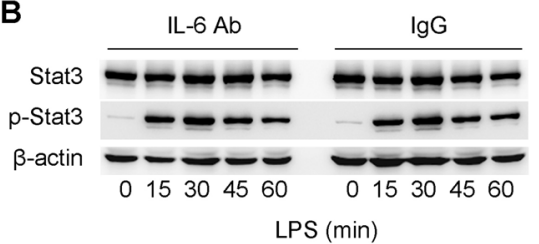

D

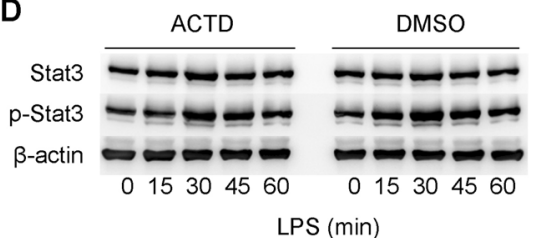

Fig. 5 LPS activates Stat3 in an IL-6 independent manner. a Primary macrophages from IL- $6^{-/-}$and WT mice were treated with LPS (100 ng/ml) for the indicated time points. p-Stat3 and Stat3 were analyzed using immunoblotting. b Primary macrophages were treated with IL- 6 antibody or mouse normal IgG and subsequently treated with LPS for the indicated time points. p-Stat 3 and Stat 3 were analyzed through immunoblotting. c-e Primary macrophages were treated with CHX, ACTD or BFA for eight hours and then stimulated with LPS (100 ng/ml) for the indicated time points. p-Stat3 and Stat3 levels were analyzed using immunoblotting. 